\title{
Tensor product of left polaroid operators
}

\author{
ENRICO BOASSO and B. P. DUGGAL
}

\begin{abstract}
A Banach space operator $T \in B(\mathcal{X})$ is left polaroid if for each $\lambda \in$ iso $\sigma_{a}(T)$ there is an integer $d(\lambda)$ such that $\operatorname{asc}(T-\lambda)=d(\lambda)<\infty$ and $(T-\lambda)^{d(\lambda)+1} \mathcal{X}$ is closed; $T$ is finitely left polaroid if $\operatorname{asc}(T-\lambda)<\infty,(T-\lambda) \mathcal{X}$ is closed and $\operatorname{dim}(T-\lambda)^{-1}(0)<\infty$ at each $\lambda \in$ iso $\sigma_{a}(T)$. The left polaroid property transfers from $A$ and $B$ to their tensor product $A \otimes B$, hence also from $A$ and $B^{*}$ to the left-right multiplication operator $\tau_{A B}$, for Hilbert space operators; an additional condition is required for Banach space operators. The finitely left polaroid property transfers from $A$ and $B$ to their tensor product $A \otimes B$ if and only if $0 \notin$ iso $\sigma_{a}(A \otimes B)$; a similar result holds for $\tau_{A B}$ for finitely left polaroid $A$ and $B^{*}$.
\end{abstract}

\section{Introduction}

A Banach space operator $T \in B(\mathcal{X})$ is polar at a point $\lambda$ in its spectrum $\sigma(T)$ if $T-\lambda I$ has both finite ascent $\operatorname{asc}(T-\lambda I)$ and descent $\operatorname{dsc}(T-\lambda I)$. Apparently, if $T$ is polar at $\lambda \in \sigma(T)$, then $\lambda \in$ iso $\sigma(T)$, the set of isolated points of $\sigma(T)$. We say that $T$ is polaroid if $T$ is polar at every $\lambda \in$ iso $\sigma(T)$. Given Banach spaces $\mathcal{X}$ and $\mathcal{Y}$, let $\mathcal{X} \bar{\otimes} \mathcal{Y}$ denote the algebraic completion, endowed with a reasonable uniform cross-norm, of the tensor product of $\mathcal{X}$ and $\mathcal{Y}$. It is known, [8, Theorem 3], that the polaroid property transfers from $A \in B(\mathcal{X})$ and $B \in B(\mathcal{Y})$ to their tensor product $A \otimes B \in B(\mathcal{X} \bar{\otimes} \mathcal{Y})$.

$T \in B(\mathcal{X})$ is left polar (respectively, right polar) of order $d$ at a point $\lambda$ in its approximate point spectrum $\sigma_{a}(T)$ (respectively, surjectivity spectrum $\sigma_{s}(T)$ ) if $\operatorname{asc}(T-\lambda I)=d<\infty$ and $(T-\lambda I)^{d+1}(\mathcal{X})$ is closed (respectively, $\operatorname{dsc}(T-\lambda I)=d<\infty$ and $(T-\lambda I)^{d} \mathcal{X}$ is closed). It is known that if $T$ is left polar (respectively, right polar) at $\lambda$, then $\lambda \in$ iso $\sigma_{a}(T)$ (respectively, $\lambda \in$ iso $\sigma_{s}(T)$ ). We say that $T$ is left polaroid (respectively, right polaroid) if $T$ is left polar (respectively, right polar) at every $\lambda \in$ iso $\sigma_{a}(T)$ (respectively, $\lambda \in$ iso $\sigma_{s}(T)$ ). Apparently, $T$ is right polaroid if and only if $T^{*}$ is left polaroid, $T$ is polaroid if it is both left and right polaroid and a polaroid operator $T$ is both left and right polaroid whenever iso $\sigma(T)=$ iso $\sigma_{a}(T) \cup$ iso $\sigma_{s}(T)$. The question that we consider here is the following: Does the left polaroid property transfer from $A \in B(\mathcal{X})$ and $B \in B(\mathcal{Y})$ to $A \otimes B \in B(\mathcal{X} \bar{\otimes} \mathcal{Y})$ ? The answer to this question is a yes in the case in which $\mathcal{X}$ and $\mathcal{Y}$ are Hilbert spaces. In the general case, if $A$ and $B$ are left polar (of order $d(\lambda)$ and $d(\mu)$ at points $\lambda \in$ iso $\sigma_{a}(A)$ and $\mu \in$ iso $\sigma_{a}(B)$ ), and if the closed subspaces $(A-\lambda I) \mathcal{X}+(A-\lambda I)^{-d(\lambda)}(0)$ and $(B-\mu I) \mathcal{Y}+(B-\mu I)^{-d(\mu)}(0)$ are complemented in $\mathcal{X}$ and $\mathcal{Y}$ respectively for every $\lambda \in$ iso $\sigma_{a}(A)$ and $\mu \in$ iso $\sigma_{a}(B)$, then $A \otimes B$ is left polaroid.

AMS Subject Classification (2010): Primary 47A80, 47A53. Secondary 47A10.

Key words and phrases: Banach space, left polaroid operator, finitely left polaroid operator, tensor product, left-right multiplication, generalized $a$-Weyl's theorem. 
A stronger version of the left polaroid property says that $T \in B(\mathcal{X})$ is finitely left polaroid if $T$ is left polar and $\alpha(T-\lambda I)=\operatorname{dim}(T-\lambda I)^{-1}(0)<\infty$ at every $\lambda \in$ iso $\sigma_{a}(T)$. The finitely left polaroid property transfers from $A \in B(\mathcal{X})$ and $B \in B(\mathcal{Y})$ to $A \otimes B$ if and only if $0 \notin$ iso $\sigma_{a}(A \otimes B)$. We characterize $\sigma_{a}(A \otimes B)$ in terms of the set of finite left poles and of the Browder essential approximate point spectrum of $A$ and of $B$, see section 4 .

Similar results will be proved for the elementary operator $\tau_{A B}=L_{A} R_{B}$ both in the case of left polaroid operators and of finitely left polaroid operators $A$ and $B^{*}$.

\section{Preliminaries}

Unless otherwise stated, from now on $\mathcal{X}$ (similarly, $\mathcal{Y}$ ) shall denote a complex Banach space and $B(\mathcal{X})$ (similarly, $B(\mathcal{Y})$ ) the algebra of all bounded linear maps defined on and with values in $\mathcal{X}$ (respectively, $\mathcal{Y}$ ). Henceforth, we shall reserve the symbols $T$ and $S$ for general Banach space operators, and the symbols $A$ and $B$ for operators $A \in B(\mathcal{X})$ and $B \in B(\mathcal{Y})$. Given $T \in B(\mathcal{X}), T^{*} \in B\left(\mathcal{X}^{*}\right)$ shall denote the adjoint of $T$, where $\mathcal{X}^{*}$ is the dual space of $\mathcal{X}$. Recall that $T \in B(\mathcal{X})$ is said to be bounded below, if $T^{-1}(0)=\{0\}$ and the range $T \mathcal{X}$ of $T$ is closed. Denote the approximate point spectrum of $T$ by $\sigma_{a}(T)=\{\lambda \in \mathbb{C}: T-\lambda$ is not bounded below $\}$, where $T-\lambda$ stands for $T-\lambda I, I$ the identity map of $B(\mathcal{X})$. Let $\sigma_{s}(T)=\{\lambda \in \mathbb{C}:(T-\lambda) \mathcal{X} \neq \mathcal{X}\}$ denote the surjectivity spectrum of $T$. Clearly, $\sigma_{a}(T) \cup \sigma_{s}(T)=\sigma(T)$, the spectrum of $T$.

Given $T \in B(\mathcal{X})$, if $T \mathcal{X}$ is closed and $\alpha(T)=\operatorname{dim} T^{-1}(0)$ (resp., $\beta(T)=\operatorname{dim} X / T \mathcal{X}$ ) is finite, then $T$ is said to be upper semi-Fredholm (resp., lower semi-Fredholm). Moreover, such an operator has a well defined index, i.e., $\operatorname{ind}(T)=\alpha(T)-\beta(T)$. Naturally, from this class of operators the upper semi-Fredholm spectrum can be derived, i. e., the set

$$
\sigma_{S F_{+}}(T)=\{\lambda \in \mathbb{C}: T-\lambda \text { is not upper semi-Fredholm }\} .
$$

The lower semi-Fredholm spectrum can be defined in a similar way and it will be denoted by $\sigma_{S F_{-}}(T)$.

Let $T \in B(\mathcal{X})$. Recall that $\operatorname{asc}(T)$ (respectively, $\operatorname{dsc}(T)$ ) is the least non-negative integer $n$ such that $T^{-n}(0)=T^{-(n+1)}(0)$ (respectively, $T^{n} \mathcal{X}=T^{n+1} \mathcal{X}$ ); if no such integer exists, then $\operatorname{asc}(T)$ (respectively, $\operatorname{dsc}(T))$ is infinite. Recall also that $\operatorname{asc}(T-\lambda)<\infty \Longrightarrow T$ has the single-valued extension property at $\lambda$, and that if $T-\lambda$ is upper semi-Fredholm and has the single-valued extension property (at 0$)$ then $\operatorname{asc}(T-\lambda)<\infty$. Here, $T$ has the single-valued extension property at $\lambda$, shortened henceforth to SVEP at $\lambda$, if, for every open neighbourhood $\mathcal{U}$ of $\lambda$, the only analytic function $f: \mathcal{U} \rightarrow \mathcal{X}$ satisfying $(T-\lambda) f(\lambda)=0$ is the function $f \equiv 0$. We say that $T$ has SVEP on a subset of the complex plane $\mathbb{C}$ if it has SVEP at every point of the subset.

The Weyl essential approximate point spectrum and the Browder essential approximate point spectrum of $T \in B(\mathcal{X})$ are the sets

$$
\sigma_{a w}(T)=\left\{\lambda \in \sigma_{a}(T): T-\lambda \text { is not upper semi-Fredholm or } 0<\operatorname{ind}(T-\lambda)\right\}
$$

and

$$
\sigma_{a b}(T)=\left\{\lambda \in \sigma_{a}(T): \lambda \in \sigma_{a w}(T) \text { or } \operatorname{asc}(T-\lambda)=\infty\right\},
$$

respectively. It is clear that

$$
\sigma_{S F_{+}}(T) \subseteq \sigma_{a w}(T) \subseteq \sigma_{a b}(T) \subseteq \sigma_{a}(T)
$$

Concerning the main properties of the aforementioned spectra, see $[1,14,15,16]$. 
We say that $T \in B(\mathcal{X})$ is semi $B$-Fredholm, $T \in \Phi_{S B F}(\mathcal{X})$, if there exists a non-negative integer $n$ such that $T^{n} \mathcal{X}$ is closed and the induced operator $T_{[n]}=\left.T\right|_{T^{n} \mathcal{X}}\left(T_{[0]}=T\right)$ is semiFredholm, upper or lower, in the usual sense. Observe that $T_{[m]}$ is then semi-Fredholm for all $m \geq n$ : we define the index of $T$ by $\operatorname{ind}(T)=\operatorname{ind}\left(T_{[n]}\right)$. Let

$$
\Phi_{S B F_{+}^{-}}(\mathcal{X})=\left\{T \in \Phi_{S B F}(\mathcal{X}): T \text { is upper semi B-Fredholm with ind }(T) \leq 0\right\} ;
$$

then the upper semi B-Weyl spectrum of $T$ is the set

$$
\sigma_{U B W}(T)=\left\{\lambda \in \sigma_{a}(T): T-\lambda \notin \Phi_{S B F_{+}^{-}}(\mathcal{X})\right\} .
$$

The lower semi B-Weyl spectrum can be defined in a similar way and it will be denoted by $\sigma_{L B W}(T)$. In addition, $T$ will be said to be $B$-Weyl, if $T$ is both upper and lower semi BFredholm (equivalently $T$ is $B$-Fredholm) and ind $(T)=0$. The $B$-Weyl spectrum of $T$ is the set

$$
\sigma_{B W}(T)=\{\lambda \in \sigma(T): T-\lambda \text { is not } B \text {-Fredholm or } \operatorname{ind}(T-\lambda) \neq 0\} .
$$

Note that $\sigma_{L B W}(T)=\sigma_{U B W}\left(T^{*}\right)$ and $\sigma_{B W}(T)=\sigma_{U B W}(T) \cup \sigma_{L B W}(T)$.

We say that $T$ is quasi-Fredholm of degree $d(\geq 0)$, if $\operatorname{dim}\left(T^{n} \mathcal{X} \cap T^{-1}(0)\right) \backslash\left(T^{n+1} \mathcal{X} \cap T^{-1}(0)\right)=$ 0 for all $n \geq d$, and the subspaces $T^{-d}(0)+T \mathcal{X}$ and $T^{-1}(0) \cap T^{d} \mathcal{X}$ are closed. Every semi BFredholm operator is quasi-Fredholm [5].

Let $\Pi^{\ell}(T)$ denote the set of left poles of $T \in B(\mathcal{X})$, i.e., $\Pi^{\ell}(T)=\left\{\lambda \in \sigma_{a}(T): \operatorname{asc}(T-\lambda)=\right.$ $d<\infty$ and $(T-\lambda)^{d+1} \mathcal{X}$ is closed $\}$. If $\lambda \in \Pi^{\ell}(T)$ is a left pole of order $d$, then $\lambda \in$ iso $\sigma_{a}(T)$, $\lambda \notin \sigma_{U B W}(T)\left(\left[9\right.\right.$, Lemma 3.1]), and $(T-\lambda)_{[d]}=\left.(T-\lambda)\right|_{(T-\lambda)^{d} \mathcal{X}}$ is bounded below $([3$, Theorem $2.5]$ ), where if $M \subseteq \mathbb{C}$, then acc $M$ stands for the set of limit points of $M$ and iso $M=M \backslash$ acc $M$. (Indeed, $\lambda \in \Pi^{\ell}(T)$ if and only if $\lambda \notin \sigma_{U B W}(T)$ and $T$ has SVEP at $\lambda$.) Furthermore, if we let

$$
H_{0}(T)=\left\{x \in \mathcal{X}: \lim _{n \longrightarrow \infty}\left\|T^{n} x\right\|^{\frac{1}{n}}=0\right\}
$$

denote the quasi-nilpotent part of $T$, then $H_{0}(T-\lambda)=(T-\lambda)^{-d}(0)([2$, Theorem 2.3]).

It is known, [9, Lemma 3.5], that if $T^{*}$ has SVEP at points $\lambda \notin \sigma_{U B W}(T)$, then $\sigma_{U B W}(T)=$ $\sigma_{B W}(T)$. This implies that if $\lambda \in \Pi^{\ell}(T)$ and $T^{*}$ has SVEP at points $\lambda \notin \sigma_{U B W}(T)$, then $\lambda \in$ iso $\sigma(T)$ and $T$ is polar at $\lambda$ ([9, Corollary 3.13]). Consequently, if $T^{*}$ has SVEP at points $\lambda \in \Pi^{\ell}(T)$, then $\Pi^{\ell}(T)=\Pi(T)=\{\lambda \in$ iso $\sigma(T): T$ is polar at $\lambda\}$.

Concerning finitely left polaroid operators, recall from [10, Theorem 3.8] that if $T \in B(\mathcal{X})$, $\alpha(T)<\infty$ and $\operatorname{asc}(T)<\infty$, then $T^{n} \mathcal{X}$ is closed for some integer $n>1$ if and only if $T \mathcal{X}$ is closed. Hence $T$ is finitely left polaroid if and only if $\alpha(T-\lambda I)<\infty, \operatorname{asc}(T-\lambda I)<\infty$ and $(T-\lambda I) \mathcal{X}$ is closed for every $\lambda \in$ iso $\sigma_{a}(T)$. Let $\Pi_{0}^{\ell}(T)$ denote the set of finite left poles of $T$, i.e.,

$$
\Pi_{0}^{\ell}(T)=\left\{\lambda \in \text { iso } \sigma_{a}(T): T-\lambda \text { is upper semi-Fredholm and } \operatorname{asc}(T-\lambda)<\infty\right\} .
$$

Then $T \in B(\mathcal{X})$ is finitely left polaroid if and only if iso $\sigma_{a}(T)=\Pi_{0}^{\ell}(T)$

In the following remark, several properties of finite left poles will be recalled.

Remark 2.1. Let $T \in B(\mathcal{X})$. Then $\sigma_{a}(T) \backslash \sigma_{a b}(T)=\Pi_{0}^{\ell}(T)$ ([16, Corollary 2.2]). Additionally, if $\lambda \in$ iso $\sigma_{a}(T)$, then the following statements are equivalent:

$$
\text { (i) } \lambda \in \sigma_{a}(T) \backslash \sigma_{a b}(T), \quad \text { (ii) } \lambda \in \sigma_{a}(T) \backslash \sigma_{a w}(T) \text {. }
$$

As a result, if we let $I_{0}^{a}(T)=$ iso $\sigma_{a}(T) \backslash \Pi_{0}^{l}(T)$, then $\left(\right.$ since $\sigma_{a w}(T) \subseteq \sigma_{a b}(T)$ ),

$$
I_{0}^{a}(T) \subseteq \sigma_{a w}(T) \subseteq I_{0}^{a}(T) \cup \operatorname{acc} \sigma_{a}(T)=\sigma_{a b}(T)
$$


([16, Theorem 2.1 and Corollary 2.3]). Therefore, necessary and sufficient for $T$ to be finitely left polaroid is that one of the following statements holds:

$$
\begin{aligned}
& \text { (iii) } \sigma_{a b}(T)=\operatorname{acc} \sigma_{a}(T), \quad\left(\text { iv) } \sigma_{a w}(T)=\operatorname{acc} \sigma_{a}(T) \cap \sigma_{a w}(T),\right. \\
& \text { (v) } \sigma_{a}(T)=\sigma_{a b}(T) \cup \text { iso } \sigma_{a}(T), \quad \sigma_{a b}(A) \cap \text { iso } \sigma_{a}(A)=\emptyset .
\end{aligned}
$$

Note that if $\sigma_{a}(T)=\{0\}$, in particular if $T$ is a quasi-nilpotent operator, then $T$ is not finitely left polaroid. In fact, if $\sigma_{a}(T)=\{0\}$ and $T$ is finitely left polaroid, then

$$
\emptyset=\operatorname{acc} \sigma_{a}(T)=\sigma_{a b}(T) .
$$

Since $\sigma_{a b}(T) \neq \emptyset([16$, Corollary 2.4] and [14, Theorem 1]), this is a contradiction.

Let $\mathcal{X} \bar{\otimes} \mathcal{Y}$ denote the completion of the algebraic tensor product of $\mathcal{X}$ and $\mathcal{Y}, \mathcal{X} \otimes \mathcal{Y}$, relative to some reasonable cross norm; let $A \otimes B \in B(\mathcal{X} \bar{\otimes} \mathcal{Y})$ denote the tensor product of $A$ and $B$. Then, [7, Lemma 5],

$$
\sigma_{a b}(A \otimes B)=\sigma_{a}(A) \sigma_{a b}(B) \cup \sigma_{a b}(A) \sigma_{a}(B) .
$$

Again, if $\tau_{A B}=L_{A} R_{B} \in B(B(\mathcal{Y}, \mathcal{X}))$ denotes the elementary operator

$$
\tau_{A B}(X)=L_{A} R_{B}(X)=A X B,
$$

then

$$
\sigma_{a b}\left(\tau_{A B}\right)=\sigma_{a}(A) \sigma_{a b}\left(B^{*}\right) \cup \sigma_{a b}(A) \sigma_{a}\left(B^{*}\right),
$$

[6, Proposition 4.3 (iv)].

The following lemma studies the sets of the limit and the isolated points of the operators considered in this article.

Lemma 2.2. If $A$ and $B$ are finitely left polaroid, then the following statements hold.

(i) acc $\sigma_{a}(A \otimes B) \subseteq \sigma_{a b}(A \otimes B) \subseteq$ acc $\sigma_{a}(A \otimes B) \cup\{0\}$;

(ii) iso $\sigma_{a}(A \otimes B) \backslash\{0\} \subseteq \Pi_{0}^{l}(A) \cdot \Pi_{0}^{l}(B) \subseteq$ iso $\sigma_{a}(A \otimes B) \cup\{0\}$.

If, instead, $A$ and $B^{*}$ are finitely left polaroid, then the following statements hold.

(iii) acc $\sigma_{a}\left(\tau_{A B}\right) \subseteq \sigma_{a b}\left(\tau_{A B}\right) \subseteq$ acc $\sigma_{a}\left(\tau_{A B}\right) \cup\{0\}$;

(iv) iso $\sigma_{a}\left(\tau_{A B}\right) \backslash\{0\} \subseteq \Pi_{0}^{l}(A) \cdot \Pi_{0}^{a}\left(B^{*}\right) \subseteq$ iso $\sigma_{a}\left(\tau_{A B}\right) \cup\{0\}$.

Proof. Since iso $\sigma_{a}(A)=\Pi_{0}^{l}(A)$ and iso $\sigma_{a}(B)=\Pi_{0}^{l}(B)$ (Remark 2.1), the proof of $(i)$ and

(ii) is immediate from [11, Theorem 6] once one observes that $\sigma_{a}(A \otimes B)=\sigma_{a}(A) \cdot \sigma_{a}(B)$ $\left(\left[12\right.\right.$, Theorem 4.4]), $\sigma_{a b}(A)=\operatorname{acc} \sigma_{a}(A), \sigma_{a b}(B)=\operatorname{acc} \sigma_{a}(B)$ (Remark 2.1), and $\sigma_{a b}(A \otimes B)=$ $\sigma_{a}(A) \sigma_{a b}(B) \cup \sigma_{a b}(A) \sigma_{a}(B)([7$, Lemma 5$])$.

One argues similarly to prove $(i i i)$ and $(i v)$ : observe that iso $\sigma_{a}(A)=\Pi_{0}^{l}(A)$ and iso $\sigma_{a}\left(B^{*}\right)=$ $\Pi_{0}^{l}\left(B^{*}\right), \sigma_{a}\left(\tau_{A B}\right)=\sigma_{a}(A) \cdot \sigma_{a}\left(B^{*}\right)([6$, Proposition $4.3(\mathrm{i})])$ and $\sigma_{a b}\left(\tau_{A B}\right)=\sigma_{a}(A) \sigma_{a b}\left(B^{*}\right) \cup$ $\sigma_{a b}(A) \sigma_{a}\left(B^{*}\right)$ ([6, Proposition 4.3 (iv) $\left.]\right)$.

Remark 2.3. Note that under the conditions of Lemma 2.2

$$
\sigma_{a b}(A \otimes B)=\sigma_{a b}(A) \cdot \sigma_{a b}(B) \cup \sigma_{a b}(A) \cdot \Pi_{0}^{l}(B) \cup \Pi_{0}^{l}(A) \cdot \sigma_{a b}(B)
$$

([7, Lemma 5]). Similarly

$$
\sigma_{a b}\left(\tau_{A B}\right)=\sigma_{a b}(A) \cdot \sigma_{a b}\left(B^{*}\right) \cup \sigma_{a b}(A) \cdot \Pi_{0}^{l}\left(B^{*}\right) \cup \Pi_{0}^{l}(A) \cdot \sigma_{a b}\left(B^{*}\right)
$$

([6, Proposition 4.3 (iv)]). 


\section{Left polaroid operators}

We say that a left polar operator $T \in B(\mathcal{X})$, of order $d(\lambda)$ at $\lambda \in$ iso $\sigma_{a}(T)$, satisfies property $(\mathcal{P})$ if the closed subspace $(T-\lambda)^{-d(\lambda)}(0)+(T-\lambda) \mathcal{X}$ is complemented in $\mathcal{X}$ for every $\lambda \in$ iso $\sigma_{a}(T)$. The following lemma proves that left polaroid operators satisfying property $\mathcal{P}$ have a Kato type decomposition.

Lemma 3.1. If $T \in B(\mathcal{X})$ is left polaroid and satisfies property $(\mathcal{P})$, then for every $\lambda \in$ iso $\sigma_{a}(T)$ there exist $T$-invariant closed subspaces $E_{1}$ and $E_{2}$ such that $\mathcal{X}=E_{1} \oplus E_{2}, H_{0}(T-\lambda)=$ $(T-\lambda)^{-d(\lambda)}(0)=H_{0}\left(\left.(T-\lambda)\right|_{E_{1}}\right)$ and $\left.(T-\lambda)\right|_{E_{2}}$ is bounded below, where $d(\lambda)$ is the order of the left pole at $\lambda$.

Proof. The hypotheses imply that $T-\lambda$ is quasi-Fredholm of order $d(\lambda)$, and the closed subspaces $(T-\lambda)^{-d(\lambda)}(0)+(T-\lambda) \mathcal{X}$ and $(T-\lambda)^{-1}(0) \cap(T-\lambda)^{d(\lambda)} \mathcal{X}$ are complemented in $\mathcal{X}$. Hence, [13, Theorem 5], there exist $T$-invariant closed subspaces $E_{1}$ and $E_{2}$ such that $\mathcal{X}=E_{1} \oplus E_{2}$, $\left.(T-\lambda)^{d(\lambda)}\right|_{E_{1}}=0$ and $\left.(T-\lambda)\right|_{E_{2}}$ is semi-regular. (Recall, [1, Page 7], that $T-\lambda$ is semi-regular if $(T-\lambda) \mathcal{X}$ is closed and $(T-\lambda)^{-n}(0) \subseteq(T-\lambda)^{m} \mathcal{X}$ for all natural numbers $m, n$.) Since $\operatorname{asc}(T-\lambda)=d(\lambda)<\infty \Longleftrightarrow(T-\lambda)^{d(\lambda)} \mathcal{X} \cap(T-\lambda)^{-n}(0)=\{0\}$ for every natural number $n$, the semi-regular operator $\left.(T-\lambda)\right|_{E_{2}}$ is injective. Hence $\left.(T-\lambda)\right|_{E_{2}}$ is bounded below. Observe that

$$
\begin{aligned}
H_{0}(T-\lambda) & =H_{0}\left(\left.(T-\lambda)\right|_{E_{1}}\right) \oplus H_{0}\left(\left.(T-\lambda)\right|_{E_{2}}\right) \\
& =E_{1} \oplus 0=E_{1} .
\end{aligned}
$$

This, since $H_{0}(T-\lambda)=(T-\lambda)^{-d(\lambda)}(0)$ by [2, Theorem 2.3], completes the proof.

Next follows the main result of this section.

Theorem 3.2. Let $A$ and $B$ be left polaroid operators. If $A$ and $B$ satisfy property $(\mathcal{P})$, or if $\mathcal{X}$ and $\mathcal{Y}$ are Hilbert spaces, then $A \otimes B$ is left polaroid.

Proof. We consider the case in which $\mathcal{X}, \mathcal{Y}$ are Banach spaces and $A, B$ satisfy property $(\mathcal{P})$; since $A, B$ automatically satisfy property $(\mathcal{P})$ in the case in which $\mathcal{X}, \mathcal{Y}$ are Hilbert spaces, the proof for the Hilbert space case is a consequence of the Banach space case.

Since $\sigma_{a}(A \otimes B)=\sigma_{a}(A) \sigma_{a}(B)$, iso $\sigma_{a}(A \otimes B)=$ iso $\left(\sigma_{a}(A) \sigma_{a}(B)\right) \subseteq$ iso $\sigma_{a}(A)$ iso $\sigma_{a}(B)$; furthermore, this is easily seen, iso $\sigma_{a}(A \otimes B) \backslash\{0\} \subseteq$ iso $\sigma_{a}(A)$ iso $\sigma_{a}(B) \subseteq$ iso $\sigma_{a}(A \otimes B) \cup\{0\}$. We consider the cases $(i) \quad 0 \neq \lambda \in$ iso $\sigma_{a}(A \otimes B)$ and $(i i) \quad 0=\lambda \in$ iso $\sigma_{a}(A \otimes B)$ separately.

$(i)$. In this case, for every $\lambda \in$ iso $\sigma_{a}(A \otimes B)$ there exist non-zero $\mu \in$ iso $\sigma_{a}(A)$ and $\nu \in$ iso $\sigma_{a}(B)$ such that $\mu \nu=\lambda$. The operator $A$ and $B$ being left polaroid operators which satisfy property $(\mathcal{P})$, there exist (by Lemma 3.1) $A$-invariant closed subspaces $M_{1}$ and $M_{2}$, and $B$ invariant closed subspaces $N_{1}$ and $N_{2}$, such that

$$
\mathcal{X}=M_{1} \oplus M_{2}, \mathcal{Y}=N_{1} \oplus N_{2} \text {, and } \mathcal{X} \bar{\otimes} \mathcal{Y}=M_{1} \bar{\otimes} N_{1} \oplus M_{1} \bar{\otimes} N_{2} \oplus M_{2} \bar{\otimes} N_{1} \oplus M_{2} \bar{\otimes} N_{2},
$$

where the closed subspaces $M_{i} \bar{\otimes} N_{j}, 1 \leq i, j \leq 2$, are $A \otimes B$-invariant and, for some integers $d_{1}, d_{2} \geq 1$,

$\left.(A-\mu I)^{d_{1}}\right|_{M_{1}}=0=(B-\nu I)^{d_{2}} \mid N_{1}$, and $\left.(A-\mu I)\right|_{M_{2}},\left.(B-\nu I)\right|_{N_{2}}$ are bounded below.

Let $d_{1}+d_{2}=d$. Then, since

$$
A \otimes B-\lambda(I \otimes I)=(A-\mu I) \otimes B+(\mu I \otimes(B-\nu I))=S+T \text { say }
$$




$$
\{A \otimes B-\lambda(I \otimes I)\}^{d}=\sum_{k=0}^{d}\left(\begin{array}{c}
d \\
k
\end{array}\right) S^{k} T^{d-k}
$$

implies that

$$
\left.\{A \otimes B-\lambda(I \otimes I)\}^{d}\right|_{M_{i} \bar{\otimes} N_{j}}=0 ; 1 \leq i, j \leq 2 \text { and } i, j \neq 2 .
$$

Furthermore, since $\mu \notin \sigma_{a}\left(\left.A\right|_{M_{2}}\right)$ and $\nu \notin \sigma_{a}\left(\left.B\right|_{N_{2}}\right), \lambda=\mu \nu \notin \sigma_{a}\left(\left.\left.A\right|_{M_{2}} \otimes B\right|_{N_{2}}\right)=\sigma_{a}(A \otimes$ $\left.\left.B\right|_{M_{2} \bar{\otimes} N_{2}}\right)$, and hence $\left.\{A \otimes B-\lambda(I \otimes I)\}\right|_{M_{2} \bar{\otimes} N_{2}}$ is bounded below. Thus $\mathcal{X} \bar{\otimes} \mathcal{Y}$ is the direct sum of two $A \otimes B$-invariant closed subspaces of $\mathcal{X} \otimes \mathcal{Y}$ such that the restriction of $A \otimes B-\lambda(I \otimes I)$ to one of them is nilpotent and its restriction to the other is bounded below. Apparently, $\operatorname{asc}(A \otimes B-\lambda(I \otimes I)) \leq d<\infty$ and $\{A \otimes B-\lambda(I \otimes I)\}^{d+1}(\mathcal{X} \bar{\otimes} \mathcal{Y})$ is closed; hence $A \otimes B$ is left polar at $\lambda$.

(ii). If $\lambda=0 \in$ iso $\sigma_{a}(A \otimes B)$, then either $(a) 0$ is not in one of $\sigma_{a}(A)$ and $\sigma_{a}(B)$, or $(b)$ $0 \in \sigma_{a}(A) \cap \sigma_{a}(B)$. If $(a)$ holds and $0 \notin \sigma_{a}(A)$, then $0 \in$ iso $\sigma_{a}(B), A$ is left invertible and there exist $B$-invariant closed subspaces $N_{1}$ and $N_{2}$ such that $\mathcal{Y}=N_{1} \oplus N_{2},\left.B\right|_{N_{1}}$ is nilpotent and $\left.B\right|_{N_{2}}$ is bounded below. Since $\mathcal{X} \bar{\otimes} \mathcal{Y}=\mathcal{X} \bar{\otimes} N_{1} \oplus \mathcal{X} \bar{\otimes} N_{2},\left.A \otimes B\right|_{\mathcal{X} \otimes N_{1}}$ is nilpotent and $\left.A \otimes B\right|_{X \bar{\otimes}} N_{2}$ is bounded below. Thus $A \otimes B$ is left polar at 0 . Since a similar argument works for the case in which $0 \in$ iso $\sigma_{a}(A)$ and $0 \notin \sigma_{a}(B)$, we are left with case $(b)$. If $0 \in \sigma_{a}(A) \cap \sigma_{a}(B)$, then either $\left(b_{1}\right) 0 \in$ iso $\sigma_{a}(A) \cap$ iso $\sigma_{b}(B)$, or $\left(b_{2}\right) 0 \in$ iso $\sigma_{a}(A) \cap \operatorname{acc} \sigma_{\mathrm{a}}(\mathrm{B})$, or $\left(b_{3}\right) 0 \in \operatorname{acc} \sigma_{\mathrm{a}}(\mathrm{A}) \cap$ iso $\sigma_{\mathrm{a}}(\mathrm{B})$. If $\left(b_{1}\right)$ holds, then we copy the argument of $(i)$ above, with $\mu=\nu=0$, to obtain $A \otimes B$ is left polar at 0 . If, instead, $\left(b_{2}\right)$ (respectively, $\left(b_{3}\right)$ ) holds, then $\sigma_{a}(A)=\{0\}$ (respectively, $\sigma_{a}(B)=\{0\}$ ), and $A$ (respectively, $B$ ) is nilpotent. This implies that $A \otimes B$ is nilpotent, hence left polaroid.

Evidently, Theorem 3.2 has a right polar analogue. Observe that if an operator $T \in B(\mathcal{X})$ is polaroid (i.e., it is both left and right polaroid), then iso $\sigma(T) \cap\left\{\sigma_{U B W}(T) \cup \sigma_{L B W}(T)\right\}=$ iso $\sigma(T) \cap \sigma_{B W}(T)=\emptyset$. In such a case, there exists an integer $d(\lambda)>0$ such that co $-\operatorname{dim}((T-$ $\left.\lambda) \mathcal{X}+(\mathrm{T}-\lambda)^{-\mathrm{d}(\lambda)}(0)\right)$ and $\operatorname{dim}\left(\left((T-\lambda)^{-1}(0) \cap(T-\lambda)^{d(\lambda)} \mathcal{X}\right)\right)$ are both finite at every $\lambda \in$ iso $\sigma(T)$. Hence there exist $T$-invariant closed subspaces $E_{1}$ and $E_{2}$ such that $\mathcal{X}=E_{1} \oplus E_{2},\left.(T-\lambda)\right|_{E_{1}}$ is $d(\lambda)$-nilpotent and $\left.(T-\lambda)\right|_{E_{2}}$ is invertible at every $\lambda \in$ iso $\sigma(T)(c f$. [13, Theorem 7]). The argument of the proof of Theorem 3.2 implies the following.

Corollary 3.3. [8, Theorem 3] $A$ and $B$ polaroid implies $A \otimes B$ polaroid.

The Hilbert space version of Theorem 3.2 has a $\tau_{A B}$ analogue.

Theorem 3.4. If $A \in B(\mathcal{H})$ and $B^{*} \in B(\mathcal{K})$ are left polaroid Hilbert space operators, then $\tau_{A B}$ is left polaroid

Proof. To prove the Theorm, one argues as in the proof of [8, Corollary 4]: $B(B(\mathcal{K}), B(\mathcal{H}))$ is an ultraprime Banach $(B(\mathcal{K}), B(\mathcal{H}))$ bimodule, and hence $\tau_{A B}$ is just $A \otimes B^{*}$. Here the ultraprime condition $\left\|L_{A} R_{B}\right\|=\|A\|\|B\|$ ensures that the operator norm of the bimodule induces a uniform cross-norm on $\mathcal{H} \bar{\otimes} \mathcal{K}$.

\section{Finitely left polaroid operators}

Recall that upper semi-Fredholm operators have a Kato decomposition: indeed, if $\lambda \notin \sigma_{a b}(T)$, then there exist $T$-invariant closed subspaces $E_{1}$ and $E_{2}$ such that $H_{0}(T-\lambda)=H_{0}\left(\left.(T-\lambda)\right|_{E_{1}}\right)=$ $(T-\lambda)^{-d}(0)$ for some integer $d=\operatorname{asc}(T-\lambda)>0, \operatorname{dim} H_{0}(T-\lambda)=\operatorname{dim} E_{1}<\infty$, and $\left.(T-\lambda)\right|_{E_{2}}$ is bounded below. Apparently, if the operators $A$ and $B$ are finitely left polaroid, then $A \otimes B$ is left polaroid. The finitely left polaroid property does not transfer from $A, B$ to $A \otimes B$; the problem, as one would expect, lies with the point 0 . 
Theorem 4.1. If $A$ and $B$ are finitely left polaroid, then $A \otimes B$ is finitely left polaroid if and only if $0 \notin$ iso $\sigma_{a}(A \otimes B)$.

Proof. Recall, [7, Lemma 5], that

$$
\sigma_{a b}(A \otimes B)=\sigma_{a b}(A) \sigma_{a}(B) \cup \sigma_{a}(A) \sigma_{a b}(B) .
$$

Suppose that $0 \neq \lambda \notin$ iso $\sigma_{a}(A \otimes B)$. Then there exist non-zero $\mu \in$ iso $\sigma_{a}(A)$ and $\nu \in$ iso $\sigma_{a}(B)$ such that $\mu \nu=\lambda$. If $A$ and $B$ are finitely left polaroid, then $\mu \notin \sigma_{a b}(A)$ and $\nu \notin \sigma_{a b}(B)$. Hence

$$
\lambda \notin \sigma_{a b}(A \otimes B) \Longleftrightarrow \lambda \in \Pi_{0}^{l}(A \otimes B) .
$$

Now let $\lambda=0$. Since the finitely left polaroid hypothesis on $A$ (respectively, $B$ ) implies that $\mathcal{X}$ (respectively, $\mathcal{Y}$ ) has a direct sum decomposition of the type considered in the proof of Theorem 3.2 whenever $0 \in$ iso $\sigma_{a}(A)$ (respectively, $0 \in$ iso $\sigma_{a}(B)$ ), it follows from the argument of the proof of Theorem 3.2, see (ii) of the proof, that $A \otimes B$ is left polaroid at 0 , with $\alpha(A \otimes B)=$ $\infty$. Hence $A \otimes B$ is not finitely left polaroid at 0 .

Remark 4.2. The upper semi-Fredholm spectrum $\sigma_{S F_{+}}(T)$ of an operator $T$ satisfies the inclusion $\sigma_{S F_{+}}(T) \subseteq \sigma_{a b}(T)$. Since $0 \notin \sigma_{a}(T) \backslash \sigma_{S F_{+}}(T)$ for every operator $T$ ([7, Lemma 4]), $0 \notin \sigma_{a}(A \otimes B) \backslash \sigma_{a b}(A \otimes B)=\Pi_{0}^{\ell}(A \otimes B)$ : this provides an alternative proof of a part of Theorem 4.1.

Remark 4.3. If the hypotheses of Theorem 4.1 are satisfied, then to prove Theorem 4.1 it is enough to consider the case $0 \in \sigma_{a}(A \otimes B)$; see Lemma 2.2(i) and Remark 2.1(iii). Observe that if $0 \notin$ iso $\sigma_{a}(A \otimes B)$, then $A \otimes B$ is finitely left polaroid (by Lemma 2.2(i) and Remark 2.1(iii)). If, instead, $A \otimes B$ is finitely left polaroid and $0 \in$ iso $\sigma_{a}(A \otimes B) \subseteq$ iso $\sigma_{a}(A)$ iso $\sigma_{a}(B)=$ $\Pi_{0}^{l}(A) \Pi_{0}^{l}(B)$, then $0 \in \Pi_{0}^{\ell}(A)$ or $0 \in \Pi_{0}^{\ell}(B)$. However, if $0 \in \Pi_{0}^{l}(A)$ (respectively, $0 \in \Pi_{0}^{\ell}(B)$ ), then, since acc $\sigma_{a}(B)=\sigma_{a b}(B) \neq \emptyset$ (respectively, acc $\sigma_{a}(A)=\sigma_{a b}(A) \neq \emptyset$ ) and $\sigma_{a}(A \otimes B)=$ $\sigma_{a}(A) \sigma_{a}(B), 0 \in$ acc $\sigma_{a}(A \otimes B)$, which is a contradiction. This provides yet another proof of Theorem 4.1.

The following remark is a supplement to the conclusions of Theorem 4.1. In fact, given $A$ and $B$ two finitely left polaroid operators, $\sigma_{a}(A \otimes B)$ will be fully described in terms of the Browder essential approximate point spectrum and the set of finite left poles of the operators $A$ and $B$. Observe that Remark 2.3 describes $\sigma_{a b}(A \otimes B)$ for finitely left polaroid operators $A$ and $B$.

Remark 4.4. Let, as in Theorem 4.1, $A$ and $B$ be two finitely left polaroid operators.

(i) If $0 \notin \sigma_{a}(A) \cdot \sigma_{a}(B)=\sigma_{a}(A \otimes B)$, then according to Theorem 4.1 and Lemma 2.2(ii), acc $\sigma_{a}(A \otimes B)=\sigma_{a b}(A \otimes B)$ and $\Pi_{0}^{l}(A \otimes B)=$ iso $\sigma_{a}(A \otimes B)=\Pi_{0}^{l}(A) \cdot \Pi_{0}^{l}(B)$. Same conclusions can be derived when $0 \in \operatorname{acc} \sigma_{a}(A) \backslash$ iso $\sigma_{a}(B)$ or $0 \in \operatorname{acc} \sigma_{a}(B) \backslash$ iso $\sigma_{a}(A)$.

(ii) If $0 \in \operatorname{acc} \sigma_{a}(A) \cap$ iso $\sigma_{a}(B)$, then according to the last observation in Remark 2.1 and Lemma 2.2(i), acc $\sigma(A \otimes B)=\sigma_{a b}(A \otimes B)$. In addition, according to Lemma 2.2(ii), iso $\sigma_{a}(A \otimes$ $B)=\Pi_{0}^{l}(A \otimes B)=\Pi_{0}^{l}(A) \cdot\left(\Pi_{0}^{l}(B) \backslash\{0\}\right)$. Similarly, if $0 \in \operatorname{acc} \sigma_{a}(B) \cap$ iso $\sigma_{a}(A)$, then iso $\left.\sigma_{a}(A \otimes B)=\Pi_{0}^{l}(A \otimes B)=\left(\Pi_{0}^{l}(A) \backslash\{0\}\right) \cdot \Pi_{0}^{l}(B)\right)$.

(iii) If $0 \in$ iso $\sigma_{a}(A)$ and $0 \notin \sigma_{a}(B)$, then since $\sigma_{a}(A) \cdot \sigma_{a}(B)=\sigma_{a}(A \otimes B)$, a standard argument on convergent subsequences proves that $0 \in$ iso $\sigma_{a}(A \otimes B)$. Consequently, according to Lemma 2.2(i)-(ii) and [7, Lemma 5], $\sigma_{a b}(A \otimes B)=\operatorname{acc} \sigma_{a}(A \otimes B) \cup\{0\}, I_{0}^{a}(A \otimes B)=\{0\}, \Pi_{0}^{l}(A \otimes B)=$ $\left(\Pi_{0}^{l}(A) \backslash\{0\}\right) \cdot \Pi_{0}^{l}(B)$, iso $\sigma_{a}(A \otimes B)=\Pi_{0}^{l}(A) \cdot \Pi_{0}^{l}(B)$ and acc $\sigma_{a}(A \otimes B)=\sigma_{a b}(A) \cdot \sigma_{a b}(B) \cup$ $\sigma_{a b}(A) \cdot \Pi_{0}^{l}(B) \cup\left(\Pi_{0}^{l}(A) \backslash\{0\}\right) \cdot \sigma_{a b}(B)$. 
(iv) If $0 \in$ iso $\sigma_{a}(A) \cap$ iso $\sigma_{a}(B)$, then an argument similar to the one in (iii) proves that iso $\sigma_{a}(A \otimes B)=\Pi_{0}^{l}(A) \cdot \Pi_{0}^{l}(B), \Pi_{0}^{l}(A \otimes B)=\left(\Pi_{0}^{l}(A) \backslash\{0\}\right) \cdot\left(\Pi_{0}^{l}(B) \backslash\{0\}\right), I_{0}^{a}(A \otimes B)=\{0\}$, $\sigma_{a b}(A \otimes B)=\operatorname{acc} \sigma_{a}(A \otimes B) \cup\{0\}$ and acc $\sigma_{a}(A \otimes B)=\sigma_{a b}(A) \cdot \sigma_{a b}(B) \cup \sigma_{a b}(A) \cdot\left(\Pi_{0}^{l}(B) \backslash\{0\}\right) \cup$ $\left(\Pi_{0}^{l}(A) \backslash\{0\}\right) \cdot \sigma_{a b}(B)$.

Note that the transfer property for finitely left polaroid operators holds in (i) and (ii).

We consider next the elementary operator $\tau_{A B}$ (where, as before, $A \in B(\mathcal{X})$ and $B \in B(\mathcal{Y})$ ).

Theorem 4.5. If $A$ and $B^{*}$ are finitely left polaroid operators, then $\tau_{A B}$ is finitely left polaroid if and only if $0 \notin$ iso $\sigma_{a}\left(\tau_{A B}\right)$.

Proof. Recall that $\sigma_{a}\left(\tau_{A B}\right)=\sigma_{a}(A) \sigma_{a}\left(B^{*}\right)$ and $\sigma_{a b}\left(\tau_{A B}\right)=\sigma_{a b}(A) \sigma_{a}\left(B^{*}\right) \cup \sigma_{a}(A) \sigma_{a b}\left(B^{*}\right)[6$, Proposition 4.1]. Now argue as in the proof of Theorem 4.1 to prove that $\tau_{A B}$ is finitely left polaroid at every non-zero $\lambda \in$ iso $\sigma_{a}\left(\tau_{A B}\right)$, and as in Remark 4.2 to prove that $\tau_{A B}$ is not finitely left polaroid at $0 \in$ iso $\sigma_{a}\left(\tau_{A B}\right)$.

Apparently, an alternative proof of Theorem 4.5 is obtained from an argument similar to the one in Remark 4.3. Furthermore, arguing just as for the operator $A \otimes B$ in Remark 4.4, it is possible to obtain a complete characterization of the sets $\sigma_{a}\left(\tau_{A B}\right)$, acc $\sigma_{a}\left(\tau_{A B}\right)$, iso $\sigma_{a}\left(\tau_{A B}\right)$, $I_{0}^{a}\left(\tau_{A B}\right)$ and $\Pi_{a}^{l}\left(\tau_{A B}\right)$, in terms of the corresponding sets for $A$ and $B^{*}$. The details are left to the reader.

We end this section by studying perturbations of finitely left polaroid operators by quasinilpotents.

If $Q_{1} \in B(\mathcal{X})$ and $Q_{2} \in B(\mathcal{Y})$ are quasi-nilpotents which commute with $A \in B(\mathcal{X})$ and $B \in$ $B(\mathcal{Y})$ respectively, then $\left(A+Q_{1}\right) \otimes\left(B+Q_{2}\right)=(A \otimes B)+Q$, where $Q=A \otimes Q_{2}+Q_{1} \otimes B+Q_{1} \otimes Q_{2}$ is a quasi-nilpotent which commutes with $A \otimes B$. Since

$$
\begin{aligned}
& \sigma_{a}\left(\left(A+Q_{1}\right) \otimes\left(B+Q_{2}\right)\right)=\sigma_{a}\left(A+Q_{1}\right) \sigma_{a}\left(B+Q_{2}\right)=\sigma_{a}(A) \sigma_{a}(B) \text { and } \\
& \sigma_{a b}\left(\left(A+Q_{1}\right) \otimes\left(B+Q_{2}\right)\right)=\sigma_{a b}\left(A+Q_{1}\right) \sigma_{a}\left(B+Q_{2}\right) \cup \sigma_{a}\left(A+Q_{1}\right) \sigma_{a b}\left(B+Q_{2}\right) \\
= & \sigma_{a b}(A) \sigma_{a}(B) \cup \sigma_{a}(A) \sigma_{a b}(B),
\end{aligned}
$$

$A$ and $B$ finitely left polaroid implies $\left(A+Q_{1}\right) \otimes\left(B+Q_{2}\right)$ finitely left polaroid at every $0 \neq$ $\lambda \in$ iso $\sigma_{a}\left(\left(A+Q_{1}\right) \otimes\left(B+Q_{2}\right)\right)$. Furthermore, since $A \otimes B=\left(A+Q_{1}\right) \otimes\left(B+Q_{2}\right)-Q, Q$ as above, we have:

Corollary 4.6. If $A \in B(\mathcal{X})$ and $B \in B(\mathcal{Y})$ are finitely left polaroid, and $Q_{1} \in B(\mathcal{X})$ and $Q_{2} \in$ $B(\mathcal{Y})$ are quasi-nilpotents which commute with $A$ and $B$ respectively, then $\left(A+Q_{1}\right) \otimes\left(B+Q_{2}\right)$ is finitely left polaroid if and only if $0 \notin$ iso $\sigma_{a}\left(\left(A+Q_{1}\right) \otimes\left(B+Q_{2}\right)\right)$.

\section{An application}

For an operator $T \in B(\mathcal{X})$, let $E^{a}(T)=\left\{\lambda \in\right.$ iso $\left.\sigma_{a}(T): 0<\alpha(T-\lambda)\right\}$ and $E_{0}^{a}(T)=\{\lambda \in$ $\left.E^{a}(T): \alpha(T-\lambda)<\infty\right\}$. Recall that $T$ is said to satisfy $a$-Browder's theorem, $a$-Bt for short (respectively, generalized $a$-Browder's theorem, $a$-gBt for short) if $\sigma_{a}(T) \backslash \sigma_{a w}(T)=\Pi_{0}^{l}(T)$ (respectively, $\sigma_{a}(T) \backslash \sigma_{U B W}(T)=\Pi^{\ell}(T)$ ). The following equivalence is well known [4, Theorem 2.2]: $T$ satisfies $a-B t$ if and only if $T$ satisfies a-gBt. $T$ satisfies $a$-Weyl's theorem, $a$-Wt for short (respectively, generalized $a$-Weyl's theorem, $a$-gWt for short) if $\sigma_{a}(T) \backslash \sigma_{a w}(T)=E_{0}^{a}(T)$ (respectively, $\sigma_{a}(T) \backslash \sigma_{U B W}(T)=E^{a}(T)$ ). The following one way implication holds: $T$ satisfies a-gWt implies $T$ satisfies $a$-Wt. Next generalized $a$-Weyl's theorem for $A \otimes B$ will be studied under the assumption $A$ and $B$ (finitely) left polaroid. 
Theorem 5.1. Suppose that $A \in B(\mathcal{X})$ and $B \in B(\mathcal{Y})$ satisfy a-Bt. If (i) $A, B$ are finitely left polaroid, or (ii) $\mathcal{X}, \mathcal{Y}$ are Hilbert spaces and $A, B$ are left polaroid, then $A \otimes B$ satisfies a-gWt if and only if $\sigma_{a w}(A \otimes B)=\sigma_{a w}(A) \sigma_{a}(B) \cup \sigma_{a}(A) \sigma_{a w}(B)$.

Proof. If $A$ and $B$ satisfy $a$-Bt, then $A \otimes B$ satisfies $a$-Bt (consequently, also $a$-gBt) if and only if $\sigma_{a w}(A \otimes B)=\sigma_{a w}(A) \sigma_{a}(B) \cup \sigma_{a}(A) \sigma_{a w}(B)\left(\left[7\right.\right.$, Theorem 1]). Thus $\sigma_{a}(A \otimes B) \backslash \sigma_{U B W}(A \otimes B)=$ $\Pi^{\ell}(A \otimes B) \subseteq E^{a}(A \otimes B)$. Since either of the hypotheses $(i)$ and $(i i)$ of the statement of the theorem implies $A \otimes B$ is left polaroid, $E^{a}(A \otimes B) \subseteq \Pi^{\ell}(A \otimes B)$. Hence $A \otimes B$ satisfies $a$-gWt. The necessity being obvious from the implications $A \otimes B$ satisfies $a$-gWt implies $A \otimes B$ satisfies $a$-gBt implies $A \otimes B$ satisfies $a$-Bt, the proof is complete.

The finite left polaroid requirement in Theorem 5.1 may be relaxed in the case in which $A^{*}$ has SVEP on $\Pi^{\ell}(A)$ and $B^{*}$ has SVEP on $\Pi^{\ell}(B)$.

Theorem 5.2. Suppose that $A \in B(\mathcal{X})$ and $B \in B(\mathcal{Y})$ satisfy a-Bt. If $A^{*}$ has $S V E P$ at points not in $\sigma_{U B W}(A), B^{*}$ has $S V E P$ at points not in $\sigma_{U B W}(B)$ and $A, B$ are left polaroid, then $A \otimes B$ satisfies a-gWt if and only if $\sigma_{a w}(A \otimes B)=\sigma_{a w}(A) \sigma_{a}(B) \cup \sigma_{a}(A) \sigma_{a w}(B)$.

Proof. The necessity follows as in the proof of Theorem 5.1; also, if $A, B$ satisfy $a$-Bt, and $\sigma_{a w}(A \otimes B)=\sigma_{a w}(A) \sigma_{a}(B) \cup \sigma_{a}(A) \sigma_{a w}(B)$, then $A \otimes B$ satisfies $a$-gBt. If $\mu \in \Pi^{\ell}(A)$, then the SVEP hypothesis on $A^{*}$ implies implies the existence of $A$-invariant subspaces $M_{1}$ and $M_{2}$ such that $\mathcal{X}=M_{1} \oplus M_{2},\left.(A-\mu)\right|_{M_{1}}$ is nilpotent and $\left.(A-\mu)\right|_{M_{2}}$ is invertible (see the argument preceding Corllary 3.3); similarly, if $\nu \in \Pi^{\ell}(B)$, then the SVEP hypothesis on $B^{*}$ implies the existence of $B$-invariant subspaces $N_{1}$ and $N_{2}$ such that $\mathcal{Y}=N_{1} \oplus N_{2},\left.(B-\nu)\right|_{N_{1}}$ is nilpotent and $\left.(B-\nu)\right|_{N_{2}}$ is invertible. The slight changes in the argument in the case in which one of $\mu$ and $\nu$ is 0 and the other is not a left pole being obvious, it follows from the argument of the proof of Theorem 3.2 that the left polaroid property transfers from $A$ and $B$ to $A \otimes B$. Hence, see the proof of Theorem 5.1, $A \otimes B$ satisfies $a-\mathrm{gWt}$.

Evidently, the operator $A \otimes B$ of Theorem 5.2 satisfies (generalized Weyl's theorem, $\sigma(A \otimes$ $B) \backslash \sigma_{B W}(A \otimes B)=E(A \otimes B)=\{\lambda \in$ iso $\sigma(A \otimes B): \lambda$ is an eigenvalue of $A \otimes B\}$ and) $a$-Wt. More is true: $\sigma_{a}(A \otimes B) \backslash \sigma_{U B W}(A \otimes B)=E(A \otimes B)$. To see this, we observe that if the hypotheses of Theorem 5.2 are satisfied, then $\Pi^{\ell}(A)=\Pi(A)=E(A), \Pi^{\ell}(B)=\Pi(B)=E(B)$ and $\sigma_{a}(A \otimes B) \backslash \sigma_{U B W}(A \otimes B)=\Pi^{\ell}(A \otimes B)=E^{a}(A \otimes B)$. Evidently, $E(A \otimes B) \subseteq E^{a}(A \otimes B)$. Let $\lambda \in E^{a}(A \otimes B)$. If $\lambda \neq 0$, then there exists $\mu \in$ iso $\sigma_{a}(A)$ and $\nu \in \sigma_{a}(B)$ such that $\mu \in \Pi^{\ell}(A)=\Pi(A) \subseteq E(A)$ and $\nu \in \Pi^{\ell}(B)=\Pi(B) \subseteq E(B)$; hence $\lambda \in E(A \otimes B)$. If, instead, $\lambda=0$, then either $0 \in \Pi^{\ell}(A) \cap \Pi^{\ell}(B)=\Pi(A) \cap \Pi(B)$, or 0 is in one of $\Pi(A), \Pi(B)$ and not in the other; in either case $0 \in E(A \otimes B)$. Hence $E(A \otimes B) \subseteq E^{a}(A \otimes B)$.

Theorem 5.1 has a $\tau_{A B}$ analogue.

Corollary 5.3. Suppose that $A \in B(\mathcal{X})$ and $B^{*} \in B\left(\mathcal{Y}^{*}\right)$ satisfy a-Bt. If (i) $A, B^{*}$ are finitely left polaroid, or (ii) $\mathcal{X}, \mathcal{Y}$ are Hilbert spaces and $A, B^{*}$ are left polaroid, then $\tau_{A B}$ satisfies a-gWt if and only if $\sigma_{a w}\left(\tau_{A B}\right)=\sigma_{a w}(A) \sigma_{a}\left(B^{*}\right) \cup \sigma_{a}(A) \sigma_{a w}\left(B^{*}\right)$.

Proof. To prove the corollary one argues as in the theorem above, using Theorem 3.4 and the fact that if $A$ and $B^{*}$ satisfy $a$-Bt then $\tau_{A B}$ satisfies $a$-gBt if and only if $\sigma_{a w}\left(\tau_{A B}\right)=$ $\sigma_{a w}(A) \sigma_{a}\left(B^{*}\right) \cup \sigma_{a}(A) \sigma_{a w}\left(B^{*}\right)([6$, Theorem 4.5]).

\section{References}

[1] P. AIENA, Fredholm and Local Spectral Theory with Applications to Multipliers, Kluwer, 2004. 
[2] P. AIENA, Quasi-Fredholm operators and localised SVEP, Acta Sci. Math. (Szeged) 73 (2007), 251-263.

[3] P. AIENA, M. T. BIONDI and C. CARPINTERO, On Drazin invertibility, Proc. Amer. Math. Soc. 136 (2008), 2839-2848.

[4] M. AMOUCH and H. ZGUITTI, On the equivalence of Browder's and generalized Browder's theorem, Glasg. Math. J. 48 (2006), 179-185.

[5] M. BERKANI and M. SARIH, On semi B-Fredholm operators, Glasg. Math. J. 43 (2001), 457-465.

[6] E. BOASSO, B. P. DUGGAL and I. H. JEON, Generalized Browder's and Weyl's theorems for left and right multiplication operators, J. Math. Anal. Appl. 370 (2010), 461-471.

[7] B. P. DUGGAL, S. V. DJORDJEVIĆ and C. S. KUBRUSLY, On the $a$-Browder and $a$-Weyl spectra of tensor products, Rend. Circ. Mat. Palermo 59 (2010), 475-483.

[8] B. P. DUGGAL, R. E. HARTE and A. H. KIM, Weyl's theorem, tensor products and multiplication operators II, Glasg. Math. J. 52 (2010), 705-709.

[9] B. P. DUGGAL, Polaroid operators and generalized Browder-Weyl theorems, Math. Proc. R. Ir. Acad. 108A (2008), 149-163 .

[10] S. GRABINER, Uniform ascent and descent of bounded operators, J. Math. Soc. Japan 34 (1982), 317-337.

[11] R. E. HARTE and A. H. KIM, Weyl's theorem, tensor products and multiplication operators, J. Math. Anal. Appl. 336 (2007), 1124-1131.

[12] T. ICHINOSE, Spectral properties of linear operators II, Trans. Amer. Math. Soc. 237 (1978), 223-254.

[13] V. MÜLLER, On the Kato decomposition of quasi-Fredholm and B-Fredholm operators, Vienna, Preprint ESI 1013 (2001).

[14] V. RAKOCEVIĆ, On one subset of M. Schechter's essential spectrum, Mat. Vesnik 5 (1981), 389-391.

[15] V. RAKOCEVIĆ, On the essential approximate point spectrum II, Mat. Vesnik 36 (1984), 89-97.

[16] V. RAKOCEVIĆ, Approximate point spectrum and commuting compact perturbations, Glasg. Math. J. 28 (1986), 193-198.

Enrico Boasso

E-mail: enrico_odisseo@yahoo.it

B. P. Duggal

E-mail: bpduggal@yahoo.co.uk 